\title{
Scapular Upward Rotator Morphologic Characteristics in Individuals With and Without Forward Head Posture: A Case-Control Study
}

Fariba Khosravi, Anneli Peolsson, Noureddin Karimi and Leila Rahnama

The self-archived postprint version of this journal article is available at Linköping University Institutional Repository (DiVA):

http:/ / urn.kb.se/ resolve?urn=urn:nbn:se:liu:diva- 154050

N.B.: When citing this work, cite the original publication.

Khosravi, F., Peolsson, A., Karimi, N., Rahnama, L., (2019), Scapular Upward Rotator Morphologic Characteristics in Individuals With and Without Forward Head Posture: A Case-Control Study,

J ournal of ultrasound in medicine, 38(2), 337-345. https:// doi.org/ 10.1002/jum.14693

Original publication available at:

https:// doi.org/ 10.1002/jum.14693

Copyright: Wiley (12 months)

http:// eu.wiley.com/WileyCDA/ 
1 Scapular upward rotators morphology in individuals with and without

2 forward head posture: a case-control study

3 Original Research

4

Fariba Khosravi, $\mathrm{MSc}^{1}$

Anneli Peolsson, $\mathrm{PhD}^{2}$

Noureddin Karimi, $\mathrm{PhD}^{1}$

Leila Rahnama, $\mathrm{PhD}^{1}$

${ }^{1}$ Department of Physiotherapy University of Social Welfare and Rehabilitation Sciences, Tehran, Iran

${ }^{2}$ Professor, Department of Medical and Health Sciences, Division of Physiotherapy, Linköping University, Linköping, Sweden

5 Corresponding Author:

6 Leila rahnama; L.rahnama@uswr.ac.ir; lrahnama@gmail.com

7 Assistant professor, Department of Physiotherapy, University of Social welfare and

8 Rehabilitation Sciences, Tehran, Iran

9 Koodakyar End Close, Daneshjou Blvd, Evin, Zip code: 1985713834, Tehran, Iran.

10 Phone: +98(21)221880084-2887

Cell phone: +98(917)1281921

11 Running Title: Scapular Upward rotators in forward head posture 


\section{Abstract}

13 Objectives: There are several reports suggesting forward head posture (FHP) contributes to

14 alteration in scapular kinematics and muscle activity leading to the development of shoulder

15 problems. Currently, it is unknown if FHP alters the thickness of the scapular muscles. The aim

16 of this study was to compare the thickness of the serratus anterior and the upper and lower

17 trapezius muscles at rest and during loaded isometric contractions in individuals with and 18 without FHP.

19 Methods: Twenty individuals with FHP and twenty individuals with normal head posture (NHP)

20 participated in this case-control study. Three separate ultrasound images of the serratus anterior

21 and the upper and lower trapezius muscles were captured under two randomized conditions: at

22 rest and during a loaded isometric contraction.

23 Results: The thickness of each muscle significantly increased from rest to the loaded isometric

24 contraction $(P<0.001)$. The only difference between the groups was that the thickness of the 25 serratus anterior muscle at rest in the NHP group was larger than in the FHP group $(P=0.01)$.

26 Conclusion: The conclusion was that FHP appears to be related to the atrophy of the serratus 27 anterior muscle, which may contribute to the development of shoulder problems. Further 28 research is required to identify more about the association of FHP with the imbalance of shoulder 29 girdle muscles and the impact of head posture on upper quadrant pain.

30 Key Words: scapular muscles, serratus anterior, trapezius, thickness, ultrasonography 
33 Faulty neck postures cause abnormal stresses on the cervical and upper thoracic structures, as 34 well as on the craniomandibular joint and the shoulder girdle. These stresses are considered to be 35 the predisposing factors of pain and disability in the upper quadrant of the body. ${ }^{1-3}$ Forward head 36 posture (FHP) is the most common faulty head posture observed in patients with neck and 37 shoulder pain. . $^{4-6}$

38 There are several shared muscle attachments between the scapula and the shoulder and axial 39 skeleton, including the upper trapezius and the levator scapulae. ${ }^{7}$ FHP may induce negative 40 effects in muscles, including muscle imbalance, not only in the neck, ${ }^{8}$ but also in the thoracic 41 spine and shoulder girdle. ${ }^{9}$ Recent research has examined the effects of head posture on the 42 scapular upward rotator muscles, which involve the serratus anterior and the upper and lower 43 trapezius muscles, and have demonstrated that there are alterations in the activity of these 44 muscles. ${ }^{1,10,11}$ Weon et $\mathrm{al}^{1}$ reported that the electromyography (EMG) activity of the trapezius 45 muscles increased, while the EMG activity in the serratus anterior muscle decreased during 46 loaded isometric shoulder flexion in individuals with simulated FHP compared with normal head 47 posture (NHP). Thigpen et $\mathrm{al}^{10}$ demonstrated that the activity of the serratus anterior muscle 48 during loaded arm flexion and overhead reaching activities decreased in individuals with FHP 49 compared with a control group.

50 Understanding the relationship between FHP and the trapezius and serratus anterior muscles may 51 provide a way to improve shoulder mechanics and decrease the risk of shoulder pain. However,

52 there is currently no knowledge about the extent of possible morphological changes that occur in 53 the scapular upward rotator muscles in individuals with FHP. Rehabilitative ultrasound imaging 54 with good clinimetric properties has been used to measure morphology, including the thickness, 
55 cross-sectional area, and muscle volume, in a variety of muscles. ${ }^{12-14}$ Furthermore, ultrasound is 56 considered to be sensitive enough to detect absolute changes in muscle thickness from rest to a 57 contracted condition. ${ }^{15}$

58 The aims of this study were to compare the thickness of the serratus anterior and the upper and 59 lower trapezius muscles at rest between individuals with and without FHP, to identify the 60 differences in the thickness of these muscles between the two groups during a loaded isometric 61 contraction, and to investigate the changes in muscle thickness from rest to a contracted 62 condition activation in each group. We hypothesized that the thickness of the scapular upward 63 rotator muscles at rest and during a loaded isometric contraction changed in participants with 64 FHP compared with participants with NHP. We also hypothesized that the thickness of these 65 muscles increased in both groups from rest to a loaded isometric contraction.

\section{Material and Methods}

\section{Participants}

68 This cross-sectional study was carried out on 20 women with FHP (aged 18-31 years) and 20

69 women with NHP (aged 18-27 years); the women with NHP acted as the control group. The 70 participants were recruited from the staff and students of University of Social Welfare and

71 Rehabilitation Sciences, Tehran, Iran. Participants were assigned to the groups based on the 72 measurement of the craniovertebral angle (CVA). Individuals with a CVA that was less than $49^{\circ}$ 73 were allocated to the FHP group; those with a CVA greater than $50^{\circ}$ were placed in the control 74 group. ${ }^{16}$ All participants were right-handed to eliminate any possible effects of handedness on 75 muscle thickness. ${ }^{1,17,18}$ Individuals were included in the study if they had a full active pain-free 76 range of motion at the neck and shoulder ${ }^{18,19}$ and a body mass index (BMI) less than 25 . The 
77 BMI was important because it was difficult to obtain clear ultrasound images of muscles covered 78 with several layers of fat.

79 Individuals were excluded from the study if they had a history of pain, injury, or surgery 80 anywhere in the neck, shoulder, or thorax that limited the range of motion of the neck or 81 shoulder such that they required time off work or a consultation with a health care 82 practitioner. ${ }^{10,11,20}$ Exclusion criteria also included structural or functional scoliosis, considerable

83 kypholordotic posture, ${ }^{10,11}$ continuous participation in sport activities, ${ }^{11}$ participation in training 84 programs that involved the scapular muscles, ${ }^{19,21}$ malignant diseases, ${ }^{17}$ pregnancy, ${ }^{19,21}$. The 85 study protocol was fully explained to all individuals, and written informed consent was obtained 86 from all participants. The study was approved by the research ethics committee of the University 87 of Social Welfare and Rehabilitation Sciences, Tehran, Iran.

\section{Procedure}

\section{Forward Head Posture Assessment}

90 Evaluation of the head posture was conducted by measuring the CVA, ${ }^{16,22}$ which is the angle 91 between the horizontal line passing through the seventh cervical spinous process (C7) and the 92 line extending from the tragus of the ear to the spinous process of C7. Digitalized lateral-view 93 photography was used to measure the CVA. The camera was placed at shoulder level, $1.5 \mathrm{~m}$ 94 from the participant's right shoulder, and positioned perpendicular to the ground. The tragus of 95 the ear was marked and a plastic pointer was attached to the skin overlying the C7 spinous 96 process, which was recognized by palpation. Participants were asked to stand in their usual 97 standing posture while looking forward and to keep their heads in a relaxed position. They were 98 then asked to perform cervical flexion and extension three times prior to standing still to achieve 
what they considered to be their relaxed head posture. Once the photograph was obtained, Adobe Photoshop CS5, Auto Desk, was used to measure the FHP, as quantified by the CVA. ${ }^{16}$

\section{Ultrasound Imaging}

102 Ultrasound images were produced using a real-time ultrasound device (Ultrasonix ES500, 103 Ultrasonix Medical Corporation, USA) with a 45-mm linear transducer in the B-mode. The 104 frequency of the imaging was set at $12 \mathrm{MHz}$ in all measurements in order to standardize the 105 measurement protocol for all participants. All imaging was conducted by the principal 106 investigator using a previously described protocol that had documented reliability in quantifying 107 the thickness of the serratus anterior and the upper and lower trapezius muscles at rest and during 108 a loaded isometric contraction..$^{19,21,23}$ The main investigator had six years of experience with 109 ultrasound imaging. Measurements of the muscle thickness were taken from the participant's 110 right side at rest and during the loaded isometric contraction while holding a 1-kg hand weight. ${ }^{17}$

111 None of the participants reported pain or discomfort during any of loaded conditions. The order 112 in which the muscles were tested was randomized in all participants.

\section{Ultrasound imaging of the serratus anterior muscle}

114 Positioning: Each participant was instructed to sit relaxed in a chair with their head and neck in 115 a relaxed position. They were then asked to place their right arm on a padded surface on an 116 adjustable bar while the shoulder was positioned in flexion of $120^{\circ}$ in the sagittal plane. The 117 position was measured with a goniometer. Images of the serratus anterior muscle were taken at 118 rest and during the loaded isometric contraction. For the rest condition, participants were 119 verbally encouraged to relax the arm being measured while keeping it in the test position. For the 120 loaded isometric contraction, participants were asked to maintain their arms in $120^{\circ}$ of the 121 shoulder flexion while holding a 1-kg hand weight for $5 \mathrm{~s}$. Verbal instruction was given to prevent 
122 participants from putting their hands on the adjustable bar. The investigator captured an image 123 during this period. Each condition was performed three times and a mean of the measurements 124 was used for data analysis.

125 Transducer location: While the participant's arm flexion was at $120^{\circ}$, the ultrasound transducer 126 was placed vertically on the midaxillary line with the superior border tangential to the horizontal 127 line passing the inferior scapular angle. The transducer was manually adjusted and tilted until the 128 echogenic borders of the ribs and the muscle fascia were well visualized. When the clear borders 129 of the serratus anterior muscle could be seen, the image was taken. The thickness of the serratus 130 anterior muscle was measured as the greatest linear distance between the hyperechoic margins of

131 the muscle over the center of the rib to the subcutaneous fascia. This method has demonstrated 132 acceptable reliability in measuring the thickness of the serratus anterior muscle. ${ }^{21,23}$ The 133 ultrasound images of the serratus anterior muscle are shown in Figure 1.

\section{$134 \quad$ Ultrasound imaging of the lower trapezius muscle}

135 Positioning: Imaging of the lower trapezius muscle was carried out at rest and during the loaded 136 isometric contraction. Participants were asked to lay prone with their heads and necks in the 137 midline. A medium-sized pillow was placed under the abdomen to eliminate any lumbar 138 hyperextension. The participant's right arm was passively moved to $120^{\circ}$ of abduction with the 139 elbow extended and the thumb pointing upward. The arm was placed on a table at the 140 appropriate angle as measured by a goniometer. For the rest condition, participants were verbally 141 encouraged to relax their arms, which were supported by the table. For the loaded isometric 142 contraction, participants were instructed to maintain their arms in the same position with no table 143 support while holding a 1-kg hand weight for $5 \mathrm{~s}$. The investigator captured an image during this 
144 period. Three separate ultrasound images of the lower trapezius muscle were taken for each 145 condition and the mean of the three measurements was used for analysis.

146 Transducer location: First, the spinous process of the eighth thoracic vertebra (T8) was 147 identified. To do this, the spinous process of the sixth cervical vertebra (C6) was palpated after 148 asking each participant to extend their neck while in a prone position. The first level above the 149 cervicothoracic junction that became less palpable was identified as the C6 spinous process. The 150 spinous processes that were inferior to C6 were then palpated and counted until the spinous 151 process of the T8 was recognized. The transducer was placed horizontally over the T8 spinous 152 process and moved laterally to the right side of T8 to observe the thickest part of the muscle in 153 way that the investigator observed the lateral border of vertebral spine on the screen. When the 154 muscle borders were clearly apparent, the image was frozen on the monitor. The thickness of the 155 lower trapezius muscle was measured as the linear distance between the two echogenic muscle

156 fascias. The reliability and validity of this procedure for measuring the lower trapezius muscle 157 have been previously established. ${ }^{19,20,23}$ The ultrasound images of the lower trapezius muscle are 158 shown in Figure 2.

\section{Ultrasound imaging of the upper trapezius muscle}

160 Positioning: Participants were asked to stand upright and to place both arms at their sides. They 161 were instructed to keep their head and neck in a neutral position. Images of the upper trapezius 162 muscle were captured at rest and during the loaded isometric contraction. Encouragement and 163 consistent verbal commands were given to the participant to relax the neck and shoulder muscles 164 when the image of the resting condition was being captured. For the loaded isometric 165 contraction, participants held a 1-kg hand weight in their right hands and were instructed to 166 perform a smooth scapular elevation of $3 \mathrm{~cm}$ in a way that their acromioclavicular joints reached 
167 an adjustable horizontal bar that was placed by their side. Participants were instructed to hold

168 this position for $5 \mathrm{~s}$. One image was frozen on the screen during $5 \mathrm{~s}$. Each condition was

169 measured three times and the average thickness measurement was used for data analysis.

170 Transducer location: To capture an ultrasound image of the upper trapezius muscle, the

171 transducer was placed at the midpoint of the line extending from the acromial tip to the spinous

172 process of C7. The linear transducer was put in a coronal plane over the landmark. Once a good

173 quality image was obtained, the image was frozen on the screen and stored. The inside edge of

174 the muscle borders was measured. The procedure for measuring the upper trapezius muscle have

175 been previously discussed. ${ }^{17,24}$ The ultrasound images of the upper trapezius muscle are shown in

176 Figure 3.

\section{Statistical Analysis}

178 Data were collected and analyzed using the Statistical Package for the Social Sciences (IBM

179 SPSS) for Windows, version 21. The demographics of the participants, including age, weight, 180 height, and BMI, were expressed as the means \pm standard deviations for both the FHP and NHP 181 groups. The mean thickness of each muscle was calculated by taking the average of the 182 measurements from the three separate trials for each of the rest and loaded isometric contraction 183 conditions in order to reduce the measurement error. The thicknesses of the muscles were 184 normalized to the individual's body weight; the normalized values were used in the statistical 185 analysis to eliminate the effects of weight on muscle size. It is recommended that normalized 186 values be used because muscle thickness is known to be affected by gender and BMI. ${ }^{25,26}$ The 187 independent t-test was used to determine any difference in demographic data between the NHP 188 and the FHP groups for each condition. Repeated measures of analysis of variance (ANOVA) 189 were used to investigate the effects of the within-group factor of contraction (rest and isometric 
contraction) and the between-group factor of head posture (FHP and NHP) on the thicknesses of the muscles. The intraclass correlation coefficient (ICC), confidence interval (CI), and standard error of measurement (SEM) were calculated for each condition and for each muscle to determine the relative and absolute reliability of the ultrasound measurements by the examiner.

\section{Results}

For this investigation, the data for 20 individuals with FHP and a mean age of $22.90 \pm 2.57$ years and 20 participants with NHP and a mean age of $23.00 \pm 3.59$ years were analyzed ( two participants were excluded from the analyzes due to suboptimal image resolution). Table 1 shows the mean and standard deviation of the demographic data of the participants in both groups. The results of the t-test showed there was no significant difference in the demographic variables between the NHP and FHP groups $(P>0.05)$.

\section{Changes in muscle thickness during the contraction and between the groups}

The group had a significant effect on the thickness of the serratus anterior muscle $(\mathrm{F}=4.55, P=$ 0.04). The between-group comparison showed that the serratus anterior muscle in the NHP group had a larger thickness at rest $(P=0.01)$ than in the FHP group, but not during the contraction $(P$ $>0.12$ ). No significant effect of group was observed for the thickness of the upper trapezius muscle $(\mathrm{F}=0.68, P=0.41)$ and the lower trapezius muscle $(\mathrm{F}=0.01, P=0.90)$, which indicated that there was no difference in the thickness of the upper and lower trapezius muscles in the FHP group compared with the NHP group at rest $(P>0.38)$ or during the loaded isometric contraction $(P>0.47)$. The loaded isometric contraction condition had a significant effect on the thickness of the serratus anterior muscle $(\mathrm{F}=25.41, P<0.001)$, the upper trapezius muscle $(\mathrm{F}=335.06, P<$ 0.001), and the lower trapezius muscle $(F=109.89, P<0.001)$ compared with the rest condition in all muscles. There was no interaction effect of group $\times$ contraction on the thickness of the 
213 serratus anterior muscle $(\mathrm{F}=0.71, P=0.40)$, the upper trapezius muscle $(\mathrm{F}=0.003, P=0.96)$, or

214 the lower trapezius muscle $(\mathrm{F}=0.09, P=0.76)$, which indicated that there was a similar rate of

215 change in the thickness of each muscle between the participants with and without FHP. The

216 mean thicknesses of the evaluated muscles are presented in Table 2.

217 The ICC and SEM values of measured muscle thickness at rest and during loaded isometric

218 contraction are presented in table 3. The intrarater reliability (ICC >0.93) was excellent for

219 serratus anterior and lower trapezius thickness measurements during rest condition and loaded

220 isometric contraction. However, the reliability of the upper trapezius thickness measurements

221 was good during loaded isometric contraction (ICC $=0.82$ ) and moderate at rest condition (ICC

$222=0.74)$.

223 Discussion

224 This study is the first study to evaluate the extent of morphological changes that occur in the 225 scapular upward rotator muscles in individuals with FHP. The results of this study demonstrated 226 that the thickness of the serratus anterior muscle in the FHP group was decreased compared with

227 the muscle in the NHP group. Based on the theoretical framework linking altered posture to 228 changes in muscle length, ${ }^{2}$ we speculated that these findings may be related to differences in the 229 muscle length between the groups. These differences are caused by the biomechanical changes 230 that take place in the cervical spine in FHP. FHP is usually associated with the shortening of the 231 posterior neck extensor muscles and the tightening of the anterior neck muscles. ${ }^{27}$ Studies have 232 reported that the levator scapulae, a cervical extensor, tends to have a short length as a postural 233 muscle with a high level of activity in individuals with FHP. ${ }^{28,29}$ Therefore, the shortening of this 234 muscle might lead to a downward rotation of the scapula. Consequently, the serratus anterior 235 muscle, as an upward rotator muscle, may be elongated when at rest. The reduced thickness of 
236 the serratus anterior muscle could result from the gradual maintenance of this elongated 237 position. ${ }^{30}$

238 However, the results of this study demonstrated that the thickness of the serratus anterior muscle 239 during the loaded isometric shoulder flexion did not significantly differ between the FHP and 240 NHP groups. It is speculated that under the contraction condition, participants with FHP may 241 require more muscle activity to act as a compensatory mechanism for the reduced muscle 242 thickness at rest. ${ }^{23,31}$ In addition, it is possible that individuals with FHP are forced to improve 243 their posture in order to complete their shoulders range of motion. Previous studies reported 244 there was a decreased activity of the serratus anterior muscle in participants with FHP compared 245 with participants with NHP. ${ }^{1,10,11}$ One possible reason for this discrepancy may be the fact that 246 EMG records the electrical activity of the muscles, while ultrasound imaging measures the 247 structural changes of the muscles. Because these parameters are different, it seems that recording 248 the electrical activity of a muscle may be more sensitive to small changes than measuring the 249 structural changes. However, there is a possibility of cross-talk and recording the electrical 250 activity from other muscles during the recording of the EMG of muscles. ${ }^{32}$ Furthermore, $^{2}$ 251 individuals with shoulder pain were excluded from the present study because pain was 252 recognized as a confounding factor due to its inhibitory effects on muscle activity. ${ }^{33}$ Therefore, 253 the decreased activity of the serratus anterior muscle observed in patients with concomitant 254 shoulder pain and FHP might have been due to the presence of pain.

255 Ultrasound measurements of the thicknesses of the upper and lower trapezius muscle at rest and 256 during a loaded isometric contraction did not appear to be significantly altered by the presence of 257 FHP. To our knowledge, this is the first study to report on the thicknesses of scapular muscles in 258 individuals with FHP using ultrasound imaging. Similar to our result, Thigpen et al ${ }^{10}$ did not find 
259 any statistically significant differences in the EMG activity of the upper and lower trapezius 260 muscles during a loaded flexion and when performing an overhead reaching task between 261 individuals with forward head and rounded shoulder posture and the control group. During 262 elevation of an arm, the trapezius muscles, together with the serratus anterior muscle, produce an 263 upward rotation, external rotation, and posterior tipping of the scapula, all of which are integral 264 to optimal scapular kinematics. ${ }^{34,35}$ Therefore, if the activity of the serratus anterior muscle is 265 altered, the activity of the trapezius muscles changes to compensate for this defect. ${ }^{36,37}$ In the 266 present study, the thickness of the serratus anterior muscle was not reduced in the FHP group 267 during the contraction condition. Thus, our results showed that there were similar amounts of 268 activity in the upper and lower trapezius muscles between the groups.

269 However, the result of the present study disagreed with studies that reported a significant 270 increase in the EMG activity of the trapezius muscle in a FHP group compared with a NHP 271 group. $^{1,11}$ This discrepancy may be attributed to differences in population, participant 272 positioning, movement patterns, external loads, and measurement techniques. For example, the 273 participants in the present study were asymptomatic individuals without shoulder pain; however, 274 other studies reported alterations in the activities of the upper and lower trapezius muscles in 275 individuals with shoulder pain $^{36}$ or in individuals with simulated $\mathrm{FHP}^{1}$. Another possible 276 explanation for the discrepancy may be the selection of movement patterns. Differences in the 277 participant's arm position between studies could contribute to variations in the position of the 278 scapula, which would affect the length of the scapular muscles when capturing an image for 279 measuring the thickness of the muscles. ${ }^{38}$ In prior studies, the EMG activities of upper and lower 280 trapezius muscles were investigated when the participant was seated with their shoulder elevated 281 in the sagittal plane ${ }^{1,11}$ while in the present study, the upper trapezius muscle was measured 
282 during elevation of the scapula in the standing position and the lower trapezius muscle was

283 measured during shoulder elevation in the frontal plane in a prone position.

284 In the present study, participants performed tasks with a 1-kg hand weight to simulate a real-life

285 activity position. In a pilot study, individuals were asked to flex their right shoulder while

286 holding a 2-kg or 1-kg hand weight and to hold the position for $5 \mathrm{~s}$. Some participants

287 complained of discomfort and fatigue in the right upper limb while holding the 2-kg hand

288 weight. Therefore, we used the 1-kg hand weight for the external load for all participants. It is

289 possible that the load in this study may not have provided an equal challenge to the upward

290 rotator muscles of all participants and, thus, may not have required high levels of recruitment of

291 the trapezius muscles. ${ }^{39}$ Future research needs to investigate possible differences in large loads.

292 Limitations

293 Our study has some limitations that should be considered when interpreting the results. It is 294 important to note that the participants were verbally encouraged to relax the arm when capturing 295 the image of the muscles at rest. However, there was no objective way to determine the 296 relaxation of these muscles. In future studies, a combination of simultaneous ultrasound and 297 EMG might provide more accurate results. ${ }^{40}$

298 In the present study, it was not possible to image more than one portion of the serratus anterior 299 and trapezius muscles at any one time. Imaging all portions of each muscle would provide a 300 better understanding of the synchronous nature of contraction. However, this method would 301 require multiple transducers and researchers and, thus, would ultimately lack clinical 302 applicability.

303 Positions used in the present study for ultrasound imaging (arm elevation in frontal plane and in 304 prone position), although recommended, may be a limitation of the present study. Elevating the 
305 arm in the sagittal or scapular planes during standing or sitting positions would be more 306 appropriate tasks because they resemble daily activity tasks.

307 There is some potential measurement error related to landmark palpations. ${ }^{41}$ Therefore. The 308 results of the presented study should be interpreted considering this potential error. However, we 309 evaluated the reliability and the standard error of measurements of the investigator 310 measurements. The results were highly reliable giving strength to the findings of the present 311 study.

312 Another limitation of the present study was that the results were not generalizable to the broad 313 population because the participants in the study were healthy, young females. It is feasible to 314 suggest that the high capability of the young participants in recruiting their muscles prevent us to 315 observe the negative impact of atrophy on the muscle contraction. ${ }^{42,43}$ Further research is

316 warranted that involves older individuals with long-term FHP to determine whether time could 317 lead to significant differences in the thickness of the scapular upward rotator muscles.

\section{Conclusion}

319 The present study revealed that FHP alters the ultrasound measurements of the thickness of the 320 serratus anterior muscle at rest. This result provided support for the clinical theory that there is a 321 relationship between FHP and atrophy of the serratus anterior muscle. Further studies are needed 322 to learn more about the relationship of FHP with the imbalance of the shoulder girdle muscles. 323 Research is also needed to assess the impact of head posture on upper quadrant pain.

324 Acknowledgments

We sincerely thank the Deputy of Research, University of Social Welfare and Rehabilitation Sciences for their financial support of the study. 


\section{References}

327 1. Weon J-H, Oh J-S, Cynn H-S, Kim Y-W, Kwon O-Y, Yi C-H. Influence of forward head posture on scapular upward rotators during isometric shoulder flexion. Journal of Bodywork and movement therapies 2010; 14(4):367-374.

330 2. Peterson-Kendall F, Kendall-McCreary E, Geise-Provance P, McIntyre-Rodgers M, Romani W. Muscles testing and function with posture and pain. 5 ed. Philadelphia, Lippincott Williams \& Wilkins; 2010.

333 3. Ayub E, Glasheen-Wray M, Kraus S. Head posture: a case study of the effects on the rest position of the mandible. Journal of Orthopaedic \& Sports Physical Therapy 1984; 5(4):179-183.

4. Chiu T, Ku W, Lee M, et al. A study on the prevalence of and risk factors for neck pain among university academic staff in Hong Kong. Journal of occupational rehabilitation 2002; 12(2):7791.

5. Haughie LJ, Fiebert IM, Roach KE. Relationship of forward head posture and cervical backward bending to neck pain. Journal of Manual \& Manipulative Therapy 1995; 3(3):91-97.

6. Braun BL, Amundson LR. Quantitative assessment of head and shoulder posture. Archives of physical medicine and rehabilitation 1989; 70(4):322-329.

342 7. Moore KL, Dalley AF, Agur AMR. Clinically Oriented Anatomy. Wolters Kluwer Health, 343 Lippincott Williams \& Wilkins; 2013.

344 8. Moghadam RE, Rahnama L, Karimi N, Amiri M, Rahnama M. An ultrasonographic investigation of deep neck flexor muscles cross-sectional area in forward and normal head posture. Journal of Bodywork and Movement Therapies 2017.

347 9. Griegel-Morris P, Larson K, Mueller-Klaus K, Oatis CA. Incidence of common postural age groups of healthy subjects. Physical therapy 1992; 72:425-425. 
10. Thigpen CA, Padua DA, Michener LA, et al. Head and shoulder posture affect scapular mechanics and muscle activity in overhead tasks. Journal of Electromyography and kinesiology 2010; 20(4):701-709.

11. Valizadeh A, Rajabi R, Rezazadeh F, Mahmoudpour A, Aali S. Comparison of the Forward Head Posture on Scapular Muscle Contributions During Shoulder Flexion of Predominant Arm in Women with Forward Head Posture. Zahedan Journal of Research in Medical Sciences 2014; 16(6):68-72.

12. BEMBEN MG. Use of diagnostic ultrasound for assessing muscle size. The Journal of Strength \& Conditioning Research 2002; 16(1):103-108.

13. Critchley DJ, Coutts FJ. Abdominal muscle function in chronic low back pain patients: measurement with real-time ultrasound scanning. Physiotherapy 2002; 88(6):322-332.

14. Esformes JI, Narici MV, Maganaris CN. Measurement of human muscle volume using ultrasonography. European journal of applied physiology 2002; 87(1):90-92.

15. Day JM, Uhl T. Thickness of the lower trapezius and serratus anterior using ultrasound imaging during a repeated arm lifting task. Manual therapy 2013; 18(6):588-593.

16. Salahzadeh Z, Maroufi N, Ahmadi A, et al. Assessment of forward head posture in females: observational and photogrammetry methods. Journal of back and musculoskeletal rehabilitation 2014; 27(2):131-139.

17. Ludvigsson ML, Peterson G, Jull G, Trygg J, Peolsson A. Mechanical properties of the trapezius during scapular elevation in people with chronic whiplash associated disorders-A case-control ultrasound speckle tracking analysis. Manual therapy 2016; 21:177-182.

18. O’Sullivan C, Persson UM, Blake C, Stokes M. Rehabilitative ultrasound measurement of trapezius muscle contractile states in people with mild shoulder pain. Manual therapy 2012; 17(2):139-144.

19. O'Sullivan C, Meaney J, Boyle G, Gormley J, Stokes M. The validity of rehabilitative ultrasound imaging for measurement of trapezius muscle thickness. Manual therapy 2009; 14(5):572-578. 
376 20. O'Sullivan C, Bentman S, Bennett K, Stokes M. Rehabilitative ultrasound imaging of the lower 377 trapezius muscle: technical description and reliability. journal of orthopaedic \& sports physical $378 \quad$ therapy 2007; 37(10):620-626.

379 21. Talbott NR, Witt DW. Ultrasound imaging of the serratus anterior muscle at rest and during contraction. Clinical physiology and functional imaging 2013; 33(3):192-200.

381 22. Nemmers TM, Miller JW, Hartman MD. Variability of the Forward Head Posture in Healthy 382 Community-dwelling Older Women. Journal of geriatric physical therapy 2009; 32(1):10-14.

383 23. Seitz AL, Baxter CJ, Benya K. Muscle thickness measurements of the lower trapezius with 384

24. Peolsson M, Larsson B, Brodin L-Å, Gerdle B. A pilot study using Tissue Velocity Ultrasound 387 Imaging (TVI) to assess muscle activity pattern in patients with chronic trapezius myalgia. BMC musculoskeletal disorders 2008; 9(1):1.

389 25. Rankin G, Stokes M, Newham D. Size and shape of the posterior neck muscles measured by 390 ultrasound imaging: normal values in males and females of different ages. Manual therapy 2005;

392 26. Springer BA, Mielcarek BJ, Nesfield TK, Teyhen DS. Relationships among lateral abdominal muscles, gender, body mass index, and hand dominance. Journal of Orthopaedic \& Sports Physical Therapy 2006; 36(5):289-297.

27. Solem-Bertoft E, Thuomas K-Å, Westerberg C-e. The influence of scapular retraction and protraction on the width of the subacromial space: an MRI study. Clinical orthopaedics and related research 1993; 296:99-103.

398 28. Janda V. Pain in the locomotor system-A broad approach. Aspects of Manipulative Therapy. Melbourne: Churchill Livingstone 1985:148-151.

400 29. McLean L. The effect of postural correction on muscle activation amplitudes recorded from the 401 cervicobrachial region. Journal of Electromyography and Kinesiology 2005; 15(6):527-535. 
30. Levangie PK, Norkin CC. Joint structure and function: a comprehensive analysis. FA Davis; 2011.

404 31. Chester R, Smith TO, Hooper L, Dixon J. The impact of subacromial impingement syndrome on

32. Morrenhof J, Abbink H. Cross-correlation and cross-talk in surface electromyography. Electromyography and clinical neurophysiology 1985; 25(1):73.

33. Lund JP, Donga R, Widmer CG, Stohler CS. The pain-adaptation model: a discussion of the relationship between chronic musculoskeletal pain and motor activity. Canadian journal of physiology and pharmacology 1991; 69(5):683-694.

412 34. Ludewig PM, Cook TM, Nawoczenski DA. Three-dimensional scapular orientation and muscle activity at selected positions of humeral elevation. Journal of Orthopaedic \& Sports Physical

415 35. Ebaugh DD, McClure PW, Karduna AR. Three-dimensional scapulothoracic motion during active and passive arm elevation. Clinical Biomechanics 2005; 20(7):700-709.

417 36. Ludewig PM, Cook TM. Alterations in shoulder kinematics and associated muscle activity in people with symptoms of shoulder impingement. Physical therapy 2000; 80(3):276-291.

419 37. Belville RG, Seupaul RA. Winged scapula in the emergency department: A case report and review. The Journal of emergency medicine 2005; 29(3):279-282.

421 38. Shi J, Zheng Y, Chen X, Xie H. Modeling the relationship between wrist angle and muscle thickness during wrist flexion-extension based on the bone-muscle lever system: A comparison

424 39. Nederhand MJ, Hermens HJ, IJzerman MJ, Turk DC, Zilvold G. Cervical muscle dysfunction in 425 27(10):1056-1061. 
427 40. Ferreira PH, Ferreira ML, Hodges PW. Changes in recruitment of the abdominal muscles in 428 people with low back pain: ultrasound measurement of muscle activity. Spine 2004; 29(22):25602566 .

430 41. Cooperstein R, Haneline M, Young M. The location of the inferior angle of the scapula in relation to the spine in the upright position: a systematic review of the literature and meta-analysis.

433 42. Lexell J, Taylor CC, Sjöström M. What is the cause of the ageing atrophy?: Total number, size 434 435 436 and proportion of different fiber types studied in whole vastus lateralis muscle from 15-to 83year-old men. Journal of the neurological sciences 1988; 84(2):275-294.

43. Young A, Stokes M, Crowe M. Size and strength of the quadriceps muscles of old and young women. European journal of clinical investigation 1984; 14(4):282-287. 
440 Table 1: Participant demographic information

\begin{tabular}{lccc}
\hline Variables & FHP(Mean \pm SD) & NHP(Mean \pm SD) & p-value \\
\hline age & $23.00 \pm 3.59$ & $22.90 \pm 2.57$ & 0.92 \\
weight & $57.07 \pm 7.17$ & $52.32 \pm 8.14$ & 0.058 \\
height & $162.97 \pm 5.21$ & $161.97 \pm 5.36$ & 0.55 \\
BMI & $21.49 \pm 2.59$ & $19.89 \pm 2.62$ & 0.06 \\
& & & \\
\hline
\end{tabular}

441

442 FHP $=$ forward head posture, $\mathrm{NHP}=$ normal head posture, $\mathrm{SD}=$ standard deviation, $\mathrm{BMI}=$ body

443 mass index

444

445 
446 Table 2. Differences in scapular muscle thickness at rest (in $\mathrm{mm}$ ) and during loaded isometric

447 contraction between groups

\begin{tabular}{llll}
\hline Muscles & condition & NHP & FHP \\
& & (Mean/weight \pm SD) & (Mean/weight \pm SD) \\
\hline Serratus anterior & rest & $0.12 \pm 0.02$ & $0.10 \pm 0.03$ \\
& contraction & $0.14 \pm 0.03$ & $0.12 \pm 0.03$ \\
lower trapezius & rest & $0.06 \pm 0.02$ & $0.07 \pm 0.02$ \\
& contraction & $0.09 \pm 0.03$ & $0.09 \pm 0.02$ \\
Upper trapezius & rest & $0.18 \pm 0.02$ & $0.17 \pm 0.04$ \\
& contraction & $0.26 \pm 0.03$ & $0.25 \pm 0.05$ \\
\hline FHP = forward head posture, NHP = normal head posture, SD = standard deviation
\end{tabular}


450 Table 3. Intraclass correlation coefficient (ICC) and standard error of the measure (SEM) for the 451 thickness measures of scapular upward rotator muscle in all participants.

452

Muscles Condition ICC (95\% CI) $\quad$ SEM

\begin{tabular}{clcc}
\hline $\begin{array}{c}\text { Serratus } \\
\text { anterior }\end{array}$ & Rest & 0.93 & 0.008 \\
& contraction & 0.95 & 0.011 \\
$\begin{array}{c}\text { lower } \\
\text { trapezius }\end{array}$ & Rest & 0.96 & 0.008 \\
& contraction & 0.96 & 0.009 \\
$\begin{array}{c}\text { Upper } \\
\text { trapezius }\end{array}$ & rest & 0.74 & 0.014 \\
& contraction & 0.82 & 0.025 \\
& & &
\end{tabular}

453

454 


\section{$455 \quad$ Figure legends}

456 Figure 1. Thickness measurement of serratus anterior in images taken at rest (a) and loaded 457 isometric contraction (b)

458 Figure 2. Thickness measurement of lower trapezius in images taken at rest (a) and during loaded 459 isometric contraction (b)

460 Figure 3. Thickness measurement of upper trapezius in images taken at rest (a) and loaded 461 isometric contraction (b) 


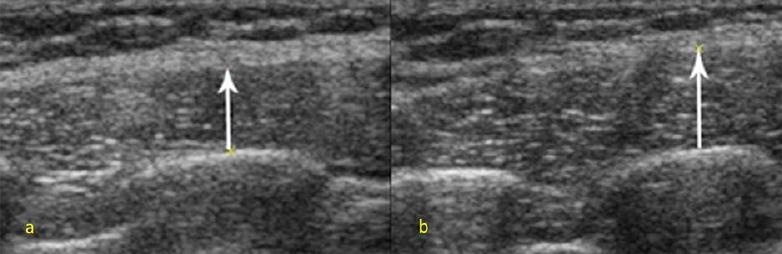




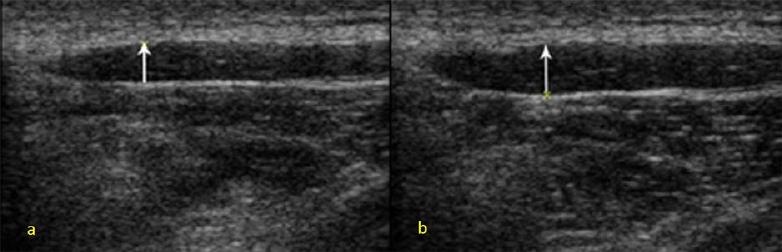




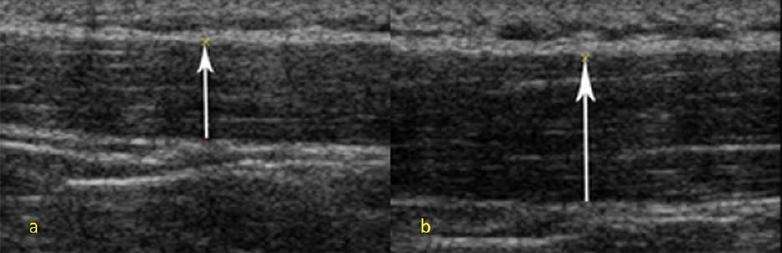

